\section{Steroid use and misuse: a key performance indicator in the management of IBD}

Steroids are an important treatment for Inflammatory Bowel Disease although have no role in maintaining remission and there are significant side effects particularly when used long term. In this issue Blackwell and colleagues discuss use and misuse. This is in the context of appropriate steroid use being regarded as a key performance indicator in the management of IBD. The paper discusses the indications for corticosteroid use, risks and strategies to reduce the use and misuse. This includes preparations and dosing regimens, an up to date discussion of toxicity and the management of side effects including how best to prevent. The authors challenge us to audit our practice which we should do and to ensure we have in place a robust team approach to the management of IBD with the early use of steroid sparing treatments where possible (see page 207).

\section{Obesity management for the gastroenterologist}

It is well known that obesity is a risk factor for adverse health outcomes short, medium and long term. In the UK approximately one in four adults are obese and one in three is overweight as defined by body mass index. Gastrointestinal and hepatic manifestations are common and can present earlier than cardiometabolic disease. This has important implications for Gastroenterologists and Hepatologists. In this issue Ansari et al provide an excellent overview in the form of a curriculum based clinical review which includes discussion of pathophysiology, medical and surgical management as well as the role of endobariatrics and the gut microbiome. There is a great deal of useful information. Table two highlights quantified risk ratios and physiological mechanisms of selected gastrointestinal diseases associated with obesity in adults which is interesting reading. Table 5 summarises NICE guidance for weight loss in adults which includes a significant reduction in calorie intake in conjunction with increased physical activity as part of a long term consistent behaviour change. Bariatric/metabolic surgery is discussed in detail including early and late complications, blood monitoring and the need for long term multivitamin and micronutrient supplementation. The article is essential reading for trainees and all those come across obesity in their clinical practice and want to impact on long term outcomes (see page 235).

\section{Specialist inflammatory bowel disease nursing in the UK: current situation and future proofing}

There are around 500000 patients with inflammatory bowel disease (IBD) in the UK. The numbers are increasing as is the complexity of case management with ever expanding treatment options. We all agree inflammatory bowel disease clinical nurse specialists (CNS) are essential to the safe and effective delivery of a quality service facilitating access, education, continuity and support. However numbers of posts remain less than recommended with not all patients having access to an IBD CNS support team. In this issue Younge et al highlight the current situation and discuss future proofing. This includes discussion of the 'More IBD Nurses - Better Care' campaign launched by Crohn's and Colitis UK in 2016 and its positive impact. The authors consider issues like recruitment and retention, training and support and set a standard which we would all like to achieve that $>95 \%$ of the charity supporters report access to an IBD specialist nurse team. We need to continue to work with our charity partners to ensure we achieve this (see page 169).

\section{IBD in pregnancy: recent advances, practical management}

There are many complexities to the management of IBD in pregnancy. In this issue Christian Selinger and colleagues discuss recent advances and practical management. Active IBD is associated with an increased risk of preterm birth, low for gestation weight and fetal loss. The clinical priority is to maintain disease remission with (mostly) the risk of a flare being greater than the risks from medication. The authors discuss a wide range of issues including fertility, pre-conception management, risk of IBD in offspring and (in detail) IBD management during pregnancy. There are useful sections on the safety of different medication, obstetric and peri-natal complications, mode of delivery, vaccination and infant feeding. It is great to have all this information in one place - up to date and authoritative. In a linked article, and with the intent to reduce variation - the team have developed standards for the provision of antenatal care for patients with inflammatory bowel disease endorsed by the British Society of Gastroenterology and the British Maternal and Foetal Medicine Society using Delphi methodology. There are 15 statements agreed by consensus. IBD and obstetric services should review their current practice against these standards. Regular audits using the proposed standards can be then performed to guide quality improvement (see pages 182 and 214).

\section{Development and evaluation of a novel protective device for upper gastrointestinal endoscopy in the COVID-19 pandemic: the EBOX}

During the initial phase of the COVID-19 pandemic there were significant constraints on endoscopy services with a virtual cessation of endoscopic activity. Although services have re started with pre procedure testing and improved patient protective equipment there is still continued uncertainty regarding the longer term impact of COVID-19 on our clinical practice. There have been many innovations. In this issue Mcleod and colleagues report the development and evaluation of a novel protective device for upper gastrointestinal endoscopy. The detail is in the paper. In summary the authors describe a new device (EBOX) for UGIE which may protect healthcare workers during the COVID-19 pandemic. They evaluate the effectiveness of the EBOX in containing droplets during a simulated cough and then evaluate the clinical utility of the EBOX in a small series of complex endoscopy procedures-ERCP and EUS. It is a really interesting read as a practical innovation with the potential to keep the endoscopy team safe during endoscopic procedures on the infected patient (see page 188).

\section{Gastroenterology 2021: from the heart of the COVID-19 pandemic}

The (ongoing) COVID-19 pandemic dominated much of our time and energies in 2020 with uncertainties, stresses, the need to change our mindset and practices but also challenges and opportunities. In this issue Oliver Tavabie and Jennie Clough 


\section{UpFront}

look back over the past year and review what we have learnt about COVID-19 and its effects on how we work as gastroenterologists as well as what questions remain unanswered. It is interesting to work through - evidence and policy emerged at an unprecedented rate and we started to think about how we would all live and work in the 'new' normal. Let's hope 2021 is better and that we embed into our practice some of the lessons learnt and innovations made (see page 249).

Please enjoy this issue. Please continue to read, enjoy and feedback on the journal. Follow us on twitter@FrontGastro_BMJ and listen to our regular podcasts accessed via the journal website https://fg.bmj.com/ 1ו||r||

Listen to Podcast

fg.bmj.com

ORCID iD

R Mark Beattie http://orcid.org/00000003-4721-0577 